\title{
Degradação Adesiva de um Compósito Auto Aderente aos Substratos da Técnica Indireta
}

\section{Bonding Degradation of a Self-Adhering Composite to Substrates used in Indirect Technique}

\author{
Rubens Nazareno Garcia ${ }^{\text {ab*; }}$ Ana Paula Coelho $;$ Francieli Althoffa; Jessica Crespia; Joel Sandri do Prado \\ anniversidade do Vale do Itajaí, Curso de Odontologia, SC, Brasil \\ bUniversidade da Região de Joinville, Departamento de Odontologia, SC, Brasil \\ *E-mail: rubensgarcia@univali.br \\ Recebido: 04 de novembro de 2015; Aceito: 15 de janeiro de 2016
} Marina Ramos Reis ; ; Monique Trierweilera; Ueslei Kohler; João Augusto Coutinho Garcia ${ }^{\mathrm{a}}$; Shélen Fernandes ${ }^{\mathrm{a}}$

\begin{abstract}
Resumo
O objetivo desse estudo foi avaliar a resistência de união - RU ao cisalhamento de um compósito fluido auto aderente em quatro substratos utilizados na técnica indireta, com uma semana e um ano de armazenamento em água destilada. Quarenta e oito amostras (5 mm largura / $15 \mathrm{~mm}$ comprimento / $2 \mathrm{~mm}$ espessura) foram preparadas em laboratório protético: 12 amostras do(a) cerômero Adoro/AD, cerâmica/leucita Empress Esthetic/EE, cerâmica/zircônia ZirCAD/ZI (Ivoclar Vivadent), mais a liga Fit Cast SB/ME (Talladium do Brasil), sendo as amostras divididas em dois grupos para cada período de tempo (uma semana e um ano). Dois compósitos fluidos (Controle / FF-Filtek Z350XT Flow / 3M ESPE e o auto aderente / DF-Dyad Flow / Kerr) foram unidos aos quatro substratos. Quatro matrizes Tygon foram posicionadas sobre as amostras, as quais foram preenchidas com os compósitos FF e DF (fotopolimerização 20s). As matrizes foram removidas para expor os corpos de prova (12 por grupo) e as amostras foram armazenadas em água destilada/ $37 \pm 2{ }^{\circ} \mathrm{C}$ por uma semana e um ano. Após estes períodos, cada amostra foi conectada a uma máquina de ensaios para realização dos ensaios de RU a uma velocidade de $1,0 \mathrm{~mm} / \mathrm{min}$, até a falha. Os resultados foram analisados por ANOVA e teste de Tukey $(\mathrm{p}<0,05)$. Nos ensaios de uma semana, os substratos leucita e metal apresentaram as maiores médias de RU para ambos os compósitos. Nos ensaios de um ano, os substratos não apresentaram diferença estatística nas médias de RU para ambos os compósitos, com exceção da zircônia que apresentou resultado nulo. Os valores médios de RU, no entanto, foram significativamente menores quando comparados com os resultados dos ensaios de uma semana.
\end{abstract}

Palavras-chave: Cerâmica. Ligas Metalo-Cerâmicas. Resinas Compostas. Resistência ao Cisalhamento.

\section{Abstract}

The aim of this study was to evaluate the shear bond strength (SBS) of a self-adhering flowable composite on four substrates used in indirect technique, for a week and one year of storage in distilled water. Forty eight samples $(5 \mathrm{~mm}$ wide $/ 15 \mathrm{~mm}$ length $/ 2 \mathrm{~mm}$ thick) were prepared at dental prosthetic laboratory: 12 samples of the ceromer Adoro/AD, leucite ceramic IPS Empress Esthetic/EE, zirconia ceramic ZirCAD/ZI (Ivoclar Vivadent) and the metal ceramic alloy Fit Cast SB/ME (Talladium do Brasil). Samples of each substrate were divided into two groups for each time (a week and one year). Two flowable composites (Control/FF-Filtek Z350 XT Flow/3M ESPE, and the self-adhering/DF-Dyad Flow/Kerr) were bonded to four substrates. Four Tygon tubing were positioned over each sample, which were filled in with the composites $F F$ and DF, and visible light-cured for 20s. The tubings were removed to expose the specimens (12 per group) in format of cylinders, and the samples were stored in distilled water at $37 \pm 2^{\circ} \mathrm{C}$ for one week and one year. After these periods, each sample was attached to testing machine and the specimens were submitted to the shear bond strength test at speed of $1.0 \mathrm{~mm} / \mathrm{min}$, until failure. The results were analyzed by ANOVA and Tukey test $(p<0.05)$. To one week tests, the substrates leucite and metal showed the highest SBS means, to both composites. For the oneyear experiments, the substrates did not show differences in the SBS averages for both composites, except for zirconia that showed null result. However, the SBS averages were lower when compared to one week tests.

Keywords: Ceramics. Metal Ceramic Alloys. Composite Resins. Shear Strength.

\section{Introdução}

A exigência dos dentistas e pacientes por restaurações na cor da estrutura dental, como inlays, onlays e coroas tem aumentado nos últimos anos e, igualmente, as técnicas para cimentação dessas restaurações. Para restaurações maiores, métodos indiretos são indicados às restaurações de resina composta direta ${ }^{1}$. Os cerômeros, cerâmicas reforçadas com leucita e zircônia, e as restaurações metalocerâmicas podem representar estes materiais, que podem ser cimentados por meio de técnica convencional e/ou adesiva. Para evitar a sensibilidade inerente à técnica adesiva, que leva aplicação de ácido fosfórico mais sistema adesivo, os cimentos resinosos autoadesivos têm sido propostos com a vantagem de ser uma técnica que pode ser feita em somente um passo para a estrutura dental; outra alternativa de cimentação pode ser um compósito fluido convencional ou auto aderente.

Os compósitos fluidos foram primeiramente introduzidos no mercado em 1995, para restaurações de lesões Classe V. Eles possuem excelentes propriedades mecânicas, baixa viscosidade e fácil manuseio, característica altamente desejada pois reduz o tempo de trabalho dos clínicos e o tempo do paciente na cadeira, de acordo com Bayne et al. ${ }^{2}$. Seguindo as mesmas características, um novo compósito fluido auto aderente, Vertise Flow (Kerr, Orange, CA, USA 
- chamado Dyad Flow na América Latina), foi recentemente introduzido no mercado, assim como o Fusio (Pentron Clinical, Orange, CA, USA) e o Constic (DMG America, Englewood, NJ, USA). Esses compósitos livres da utilização prévia de um sistema adesivo são requeridos por contar com interação química e micromecânica entre o material e a estrutura dental, ou outros substratos, alcançadas com a incorporação de um monômero adesivo ácido nos compósitos fluidos ${ }^{3-7}$, como o glicerol fosfato dimetacrilato, no caso do Dyad Flow.

Devido à novidade desse tipo de material (auto aderente), é pertinente investigar sobre a resistência de união do compósito fluido Dyad Flow. O objetivo deste estudo foi avaliar a resistência de união ao cisalhamento de um compósito fluido auto aderente a quatro substratos usados na técnica indireta, com uma semana e um ano de armazenamento em água destilada. A hipótese nula testada foi que as resistências de união do compósito fluido auto aderente seriam estatisticamente similares quando comparados os tempos de armazenamento em água destilada.

\section{Material e Métodos}

Quarenta e oito amostras $(5 \mathrm{~mm}$ de largura, $15 \mathrm{~mm}$ de comprimento e $2 \mathrm{~mm}$ de espessura / doze blocos para cada substrato) foram obtidos em laboratório protético, de acordo com as instruções do fabricante. Os blocos do cerômero SR Adoro/AD (Lote n. R57506/Val: 07/2015) foram preparados e fotopolimerizados no forno Lumamat 100 Light Furnace (Ivoclar Vivadent, Schaan, Principado de
Liechtenstein); os blocos da cerâmica reforcada com leucita IPS Empress Esthetic/EE (Lote n. KM0305/Val: 12/2030) foram preparados pela técnica da cerâmica injetada; os blocos da cerâmica reforçada com zircônia IPS e.max ZirCAD/ZI (Lote n. L15418/Val: 12/2030) foram preparados na técnica CAD/CAM (Computer-Aided Desing/Computer-Aided Manufacturing). Todos os materiais forma provenientes da IvoclarVivadent, Schaan, Principado de Liechtenstein. Os blocos do metal da liga metalocerâmica Fit Cast SB/ME (Lote n.121165/Val: indeterminado/Talladium do Brasil, Curitiba, PR, Brasil) foram preparados com a técnica da cera perdida. Todas as amostras foram jateadas com óxido de alumínio (90 $\mu \mathrm{m} / 2,5$ bar/10 mm distância) e as amostras de cada substrato foram divididas em dois grupos: compósito controle e compósito auto aderente $(\mathrm{n}=3)$, e foram novamente divididas em outros dois grupos, para os ensaios de uma semana e de um ano.

Para os grupos AD e EE, após a aplicação de ácido fluorídrico 10\% por 1 min (Condac Procelana, FGM, Joinville, SC, Brasil - Batch\#/ Exp: 12/2013), as amostras foram lavadas por $1 \mathrm{~min}$, secas por $1 \mathrm{~min}$, seguido da aplicação do silano (Monobond-S / Ivoclar Vivadent - Lote n. N15219) durante 1 min e em seguida secas com jato de ar por 30s. Para os grupos ZI e ME, foi aplicado o primer (Metal/ZirconiaPrimer / IvoclarVivadent - Lote n. M68692) por 3 min, e em seguida realizada secagem por $30 \mathrm{~s}$.

Dois compósitos fluidos (controle / FF-Filtek Z350XT Flow / 3M ESPE e o auto aderente / DF - Dyad Flow / Kerr) foram unidos aos quatro substratos (Quadro 1).

Quadro 1: Materiais utilizados

\begin{tabular}{|c|c|c|c|}
\hline Materiais & Lote & Composições & Protocolos \\
\hline $\begin{array}{l}\text { Adper Single Bond } 2 \text { Dental Adhesive } \\
\mathrm{pH} \approx 4.7 \\
3 \mathrm{M} \text { ESPE - St. Paul, MN, USA }\end{array}$ & N2I1104BR & $\begin{array}{l}\text { Bis-GMA, HEMA, UDMA, dimetacrilatos, } \\
\text { etanol, água, canforquinona, fotoiniciadores, } \\
\text { copolímero do ácido polialcenóico, sílica }(5 \mathrm{~nm})\end{array}$ & $\begin{array}{l}\text { Aplicar o adesivo, ar } 5 \mathrm{~s}, \\
\text { foto } 10 \mathrm{~s}\end{array}$ \\
\hline $\begin{array}{l}\text { Filtek Z350 XT Flow VLC Flowable } \\
\text { Nanocomposite/A2 } \\
\text { 3M ESPE - St. Paul, MN, USA }\end{array}$ & 1211700713 & $\begin{array}{l}\text { Bis-GMA, TEGDMA, Bis-EMA, cerâmica } \\
\text { tratada com silano, sílica, óxido de zircônio - } 55 \\
\% \text { vol / } 65 \% \text { peso }\end{array}$ & Aplicar e foto $20 \mathrm{~s}$ \\
\hline $\begin{array}{l}\text { Dyad Flow Self-Adhesive Flowable } \\
\text { Nanocomposite/A2 } \\
\text { pH } \approx 1.9 \text { antes foto } \\
\text { pH } \approx 6.5-7 \text { após foto } \\
\text { Kerr, Orange, CA, USA }\end{array}$ & 4398621 & $\begin{array}{l}\text { GPDM, partículas pré-polimerizadas, partículas } \\
\text { de vidro de bário com } 1-\mu \mathrm{m} \text {, nanopartículas } \\
\text { de sílica coloidal, fluoreto de itérbium } \\
\text { nanoparticulado }\end{array}$ & $\begin{array}{l}\text { Aplicar, esfregar uma fina } \\
\text { camada }(<0,5 \mathrm{~mm}) \text { com } \\
\text { pressão por } 15-20 \mathrm{~s} \text {, foto } 20 \mathrm{~s}\end{array}$ \\
\hline
\end{tabular}

Composições conforme fabricantes: Bis-GMA, bisphenol glycidyl dimethacrylate; HEMA, 2-hydroxyethyl methacrylate; UDMA, urethane dimethacrylate; TEGDMA, triethylene glycol dimethacrylate; Bis-EMA, bisphenol A polyethylene glycol dimethacrylate; GPDM, glycerol phosphate dimethacrylate.

Fonte: Dados da pesquisa.

De acordo com Shimada et al. ${ }^{1}$, para todos os grupos e em cada uma das amostras, quatro matrizes cilindrícas transparentes do tipo Tygon (Tygon Tubing - TYG-030, Saint-Gobain Performance Plastic, Maime Lakes, FL, USA) foram posicionadas sobre as amostras, que foram preenchidas com o compósitos FF e DF, e fotopolimerizadas por 20s (LED Bluephase - $1.200 \mathrm{~mW} / \mathrm{cm}^{2}$ - Ivoclar Vivadent, Schaan,
Principado de Liechtenstein). As matrizes foram removidas para expor os corpos de prova no formato cilíndrico (12 por grupo - área: $0,38 \mathrm{~mm}^{2} /$ pela fórmula $\pi \mathrm{R}^{2}$ ), e as amostras foram armazenadas em água destilada a $37 \pm 2{ }^{\circ} \mathrm{C}$ durante uma semana e um ano, com troca quinzenal da água destilada. Decorrido este período, cada amostra foi conectada à máquina de ensaios universal (Emic DL 1000, São José dos Pinhais, 
Pr, Brasil) e os corpos de prova submetidos ao ensaio de resistência de união ao cisalhamento, aplicado na base do corpo de prova cilíndrico com um fio ortodôntico fino / 0,25 $\mathrm{mm}$, a uma velocidade de $1 \mathrm{~mm} / \mathrm{min}$ - até a fratura (Figura 1).

Figura 1: Representação esquemática da metodologia empregada

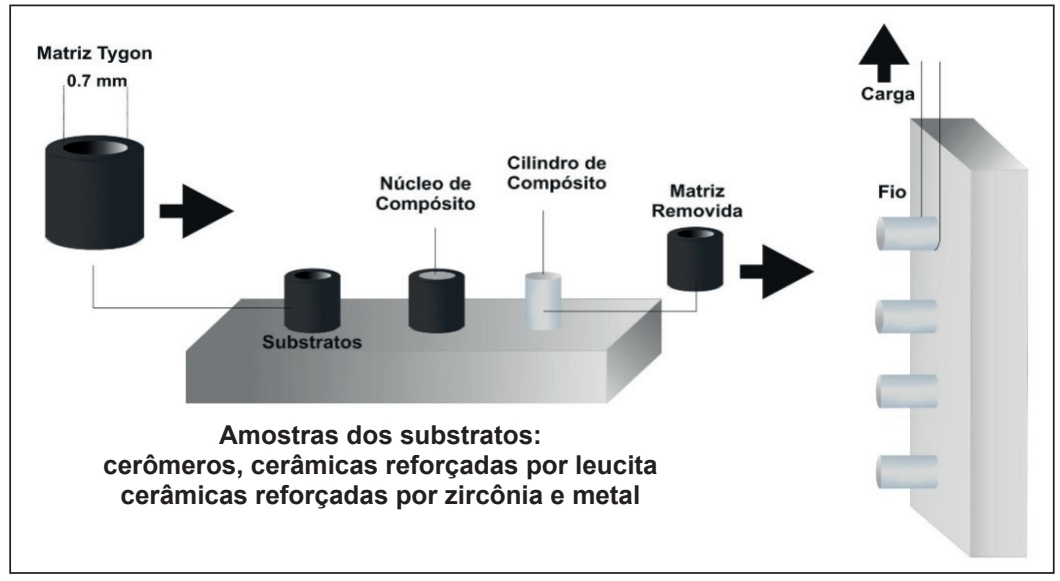

Fonte: $\mathrm{O}$ autor.

Foi utilizada microscopia ótica para avaliação das fraturas/falhas, que foram classificadas em adesivas, mistas (adesivas e coesivas) e coesivas nos substratos. Os resultados foram analisados por Análise de Variância (ANOVA) três fatores e pelo teste de Tukey $(p<0,05)$, individualmente nos tempos de uma semana e um ano, e finalmente feita a análise conjunta dos dados nos dois tempos de armazenamento.

\section{Resultados e Discussão}

A ANOVA mostrou diferenças significativas entre os compósitos fluidos, substratos e tempos e para identificar as diferenças foi aplicado o teste de Tukey $(\mathrm{p}<0,05)$ individualmente em cada tempo (uma semana / um ano), seguido de análise conjunta dos dados. Nos ensaios de uma semana, a cerâmica com leucita e o metal apresentaram as maiores médias de resistência de união e sem diferença estatística entre si para ambos os compósitos, mas com diferença significativa para o cerômero, que apresentou média intermediária de resistência de união. A cerâmica com zircônia apresentou a menor média de resistência de união (Tabela 1).

Tabela 1: Médias de RU ( \pm DP) em MPa e Teste de Tukey $(\mathrm{p}<0,05)$ - uma semana

\begin{tabular}{lcc}
\hline \multirow{2}{*}{ Uma semana } & \multicolumn{2}{c}{ Compósito } \\
\cline { 2 - 3 } & Filtek Z350 XT Flow & Dyad Flow \\
\hline Cerômero & $34,5 \pm 5,0 \mathrm{~A} \mathrm{a}$ & $28,2 \pm 4,3 \mathrm{~B} \mathrm{~b}$ \\
Leucita & $29,7 \pm 5,8 \mathrm{~A} \mathrm{~b}$ & $32,4 \pm 6,9 \mathrm{~A} \mathrm{a}$ \\
Zircônia & $23,2 \pm 5,4 \mathrm{~A} \mathrm{c}$ & $08,5 \pm 1,6 \mathrm{~B} \mathrm{c}$ \\
Metal & $28,9 \pm 4,3 \mathrm{~A} \mathrm{~b}$ & $31,8 \pm 4,5 \mathrm{~A} \mathrm{a}$ \\
\hline
\end{tabular}

ANO 1: Médias seguidas da mesma letra maiúscula nas linhas e da mesma letra minúscula nas colunas não diferem significativamente entre si pelo teste de Tukey $(\mathrm{p}<0,05)$.

Fonte: Dados da pesquisa.
Nos ensaios de um ano, não houve diferença entre os substratos para ambos os compósitos, com exceção da cerâmica com zircônia que apresentou resultado nulo (Tabela 2). Os valores de resistência de união, no entanto, foram significativamente menores quando comparados com os ensaios de uma semana e um ano (Tabela 3).

Tabela 2: Médias de RU $( \pm \mathrm{DP})$ em MPa e Teste de Tukey $(\mathrm{p}<0,05)-$ um ano

\begin{tabular}{lcl}
\hline \multirow{2}{*}{ Um ano } & \multicolumn{2}{c}{ Compósito } \\
\cline { 2 - 3 } & Filtek Z350 XT Flow & \multicolumn{1}{c}{ Dyad Flow } \\
\hline Cerômero & $23,0 \pm 5,6 \mathrm{~A} \mathrm{a}$ & $20,1 \pm 7,6 \mathrm{~A} \mathrm{a}$ \\
Leucita & $24,0 \pm 6,7 \mathrm{~A} \mathrm{a}$ & $19,4 \pm 6,0 \mathrm{~A} \mathrm{a}$ \\
Zircônia & $00,0 \pm 0,0$ & $00,0 \pm 0,0$ \\
Metal & $13,6 \pm 4,4 \mathrm{~A} \mathrm{~b}$ & $17,9 \pm 3,8 \mathrm{~A} \mathrm{a}$ \\
\hline
\end{tabular}

ANO 2: Médias seguidas da mesma letra maiúscula nas linhas e da mesma letra minúscula nas colunas não diferem significativamente entre si pelo teste de Tukey $(\mathrm{p}<0,05)$

Fonte: Dados da pesquisa.

Tabela 3: Médias de RU $( \pm \mathrm{DP})$ em MPa e Teste de Tukey $(\mathrm{p}<0,05)-\operatorname{nos} 2$ tempos

\begin{tabular}{|c|c|c|c|c|}
\hline & \multicolumn{2}{|c|}{$\begin{array}{c}\text { Compósito Filtek Z350 XT } \\
\text { Flow }\end{array}$} & \multicolumn{2}{|c|}{ Compósito Dyad Flow } \\
\hline & Uma semana & Um ano & Uma semana & Um ano \\
\hline Cerômero & $34,5 \pm 5,0 \mathrm{~A}$ & $23,0 \pm 5,6 \mathrm{~B}$ & $28,2 \pm 4,3 \mathrm{~A}$ & $20,1 \pm 7,6 \mathrm{~B}$ \\
\hline Leucita & $29,7 \pm 5,8 \mathrm{~A}$ & $24,0 \pm 6,7 \mathrm{~B}$ & $32,4 \pm 6,9 \mathrm{~A}$ & $19,4 \pm 6,0 \mathrm{~B}$ \\
\hline Zircônia & $23,2 \pm 5,4$ & $00,0 \pm 0,0$ & $08,5 \pm 1,6$ & $00,0 \pm 0,0$ \\
\hline Metal & $28,9 \pm 4,3 \mathrm{~A}$ & $13,6 \pm 4,4 \mathrm{~B}$ & $31,8 \pm 4,5 \mathrm{~A}$ & $17,9 \pm 3,8 \mathrm{~B}$ \\
\hline
\end{tabular}

Médias seguidas da mesma letra maiúscula nas linhas, dentro de cada produto, não diferem significativamente entre si pelo teste de Tukey $(\mathrm{p}<0,05)$

Fonte: Dados da pesquisa. 
Baseado nos achados do presente estudo, a hipótese nula formulada deve ser rejeitada, uma vez que os resultados mostraram diferenças estatisticamente significativas nas resistências de união em ambos os tempos testados.

Os testes laboratoriais são utilizados porque produzem informações imediatas sobre a longevidade e degradação dos materiais. Especialmente os ensaios de resistência de união fornecem uma avaliação quantitativa da adesão dos materiais, com base no conceito de quanto mais forte a ligação, melhor a resistência à contração de polimerização e às tensões funcionais ${ }^{8}$.

O presente estudo focou a avaliação da resistência de união ao cisalhamento do compósito fluido auto aderente Dyad Flow em quatro substratos da técnica indireta, utilizando a metodologia do micro cisalhamento proposta por Shimada et al. ${ }^{1}$ com um ano de armazenamento em água destilada. Este tipo de ensaio mecânico soluciona problemas relacionados à propagação de tensões do material na interface de união que ocorre em áreas maiores. A vantagem do método é que vários corpos de prova podem ser obtidos a partir de uma amostra sem cortá-la, sendo mais fácil e mais barato do que o teste de microtração9 .

Restaurações indiretas têm sido utilizadas para minimizar a contração de polimerização dos compósitos restauradores que ocorrem na técnica direta, provavelmente devida a pequena quantidade de cimento resinoso utilizado nos procedimentos de cimentação. Esta técnica proporciona melhor selamento quando comparada ao uso de compósitos diretos. Além disso, é usada para facilitar a reprodução da anatomia dentária, a fim de melhorar o controle da adaptação marginal, proximal e dos contatos oclusais. Em relação ao cerômero, quando comparado às outras restaurações indiretas, como a cerâmica, apresenta uma técnica de fabricação mais simples, menor desgaste dos dentes antagonistas, a possibilidade de reparação intraoral e menor custo. No que se referem às cerâmicas, estas são usadas para restaurações estéticas por causa da sua cor superior, resistência à compressão, condutibilidade térmica, semelhança aos tecidos dentais, radiopacidade, integridade marginal, estabilidade de cor, biomimetismo, entre outras, e seu sucesso clínico é fortemente determinado pela resistência e durabilidade da união do cimento resinoso ao dente e à cerâmica $^{10}$.

A aplicação dos cimentos resinosos em restaurações indiretas tem aumentado consideravelmente devida sua capacidade de ajudar na definição da cor do dente e a sua maior resistência às cargas oclusais, quando comparado com a cimentação convencional. Esta cimentação geralmente requer várias etapas para garantir ótima união. No entanto, recentemente, alguns primers auto condicionantes e cimentos resinosos autoadesivos foram introduzidos, gerando grandes melhorias na união às estruturas dentárias. A utilização destes produtos é o resultado de tentativas para melhorar a qualidade da união, enquanto reduz o número de procedimentos operatórios ${ }^{1}$. Para este estudo, foi proposto que o uso de um compósito fluido auto aderente poderia resultar em similar resistência de união, embora o grupo controle tenha sido também um compósito fluido, e não um cimento resinoso.

Primeiramente, as amostras de cerômero e cerâmica reforçada por leucita foram jateadas com óxido de alumínio. O jateamento da superfície interior desses materiais é uma prática comum em laminados e coroas, pois a superfície rugosa permite uma forte ligação mecânica aos materiais resinosos ${ }^{11}$. Depois disso, de acordo com as instruções do fabricante, o cerômero e a cerâmica reforçada com leucita foram condicionados com ácido fluorídrico $10 \%$ e feita a silanização. A melhor maneira de condicionamento dessas superfícies é por condicionamento com ácido fluorídrico, seguido da aplicação de um agente de união (silano) para conseguir interação química e uma elevada resistência de união. $\mathrm{O}$ ácido funciona criando ranhuras superficiais através de dissolução preferencial da fase vítrea da matriz de cerâmica e a dissolução da matriz da resina ${ }^{12}$ (no caso dos cerômeros). O tratamento da superfície com aplicação de silano aumenta a molhabilidade e forma uma ligação covalente ${ }^{13}$. O trimetoxisilano, ou simplesmente silano, é conhecido por promover adesão através de interação química e física entre compósitos e metal, compósitos e cerâmicas, e compósitos à base de metacrilato.

Três mecanismos de interação química são possíveis para a resistência de união do compósito fluido a outro compósito, o cerômero, de acordo com os achados de Teixeira et al. ${ }^{14}:$ 1) aderência entre os polímeros da matriz, de ambos, compósito fluido e cerômero; 2) a adesão entre as partículas de carga expostas de ambos os compósitos; e 3) formação de uma micro rede de cadeias poliméricas e as partículas de carga de ambos os compósitos. Provavelmente este último mecanismo é dominante e produz a maior contribuição no que diz respeito a uma resistência de união aceitável. No entanto, o substrato cerômero, para ambos os compósitos fluidos e no tempo de um ano (Tabela 3), apresentou menor resistência de união, com diferença estatisticamente significativa. Provavelmente os componentes do compósito FF e especialmente do DF (que tem propriedades de hidrofilicidade que não interessam ao cerômero) promovem uma união não estável no substrato cerômero com o passar de um ano em água destilada. Ainda que em substratos dentais, mas podendo fazer uma correlação com a questão da degradação, Garcia et al. ${ }^{15}$ relataram que as camadas híbridas de alguns adesivos auto condicionantes exibiam canais microscópicos preenchidos por água, que permitiam o movimento dessa água na interface dos substratos, e por consequência o decréscimo da união. A redução da resistência de união, nesse caso, poderia ser atribuída à degradação do compósito no meio aquoso, pelo processo de cisão da cadeia polimérica, quando a cadeia é quebrada em segmentos menores, chamados de oligômeros.

Utilizando a metodologia de microcisalhamento e o Dyad Flow, com degradação de um ano em cerômeros e cerâmicas, não há comparação com a literatura até o momento, apenas 
os dados internos do fabricante Kerr, relatados no Boletim Técnico Kerr/35104 (2010). Nesse boletim, a cerâmica descrita apresentou 33,9 $\mathrm{MPa}$ e neste estudo 32,4 $\mathrm{MPa}$ para a cerâmica reforçada com leucita (uma semana), mas o fabricante não utilizou ácido fluorídrico e silano. Os resultados de Garcia et al. ${ }^{16}$ mostraram resistência de união semelhante à cerâmica reforcada com leucita, mas os autores utilizaram cimentos resinosos, o RelyX ARC e RelyX U100, ambos da 3M ESPE. No presente estudo, quando as cerâmicas reforçadas por leucita foram utilizadas com os compósitos FF e DF, apresentaram menor resistência de união com um ano de armazenamento em água destilada.

IPS e.max ZirCAD é um bloco de óxido de zircônia estabilizado com ítrio. É apropriado para situações clínicas que requerem elevada resistência, incluindo próteses fixas posteriores. A composição e as propriedades físicas dos produtos cerâmicos à base de óxido de zircônia diferem substancialmente daqueles baseados em cerâmica com sílica, de tal modo que o condicionamento ácido convencional não tem efeito positivo sobre a cerâmica à base de óxido de zircônia $^{17}$. A união à cerâmica de alta resistência é menos previsível, e são necessárias técnicas de união alternativas para alcançar uma união forte e durável em longo prazo. Os produtos que contêm o ácido fosfônico como ingrediente ativo podem estabelecer uma ligação química nessas superfícies, tal como o Metal/Zirconia Primer. Assim, eles podem ser uma alternativa para promover a adesão à cerâmica. Neste estudo, no entanto, o produto não foi suficiente para resultar uma aceitável resistência de união para o FF e DF e a cerâmica reforçada por zircônia, especialmente no tempo de um ano, que apresentou resultado nulo. Especula-se que o sistema adesivo Adper Single Bond 2 utilizado antes do compósito fluido FF do grupo controle (uma semana) ajudou a molhabilidade do substrato cerâmica reforçada com zircônia, o que não foi observado no período de um ano.

A restauração metálica tem uma longa história de uso clinico em Odontologia. No entanto, o aumento das expectativas e exigências estéticas dos pacientes fez com que as restaurações puramente metálicas fossem parcialmente deixadas de lado, levando ao desenvolvimento das restaurações metalocerâmicas. Apesar da natureza frágil da porcelana, as coroas metalocerâmicas são amplamente utilizadas porque as estruturas metálicas promovem excelente durabilidade mecânica. Por outro lado, os usos de metais nessas restaurações também promovem a descoloração gengival e alergias relacionadas com os metais ${ }^{18}$.

A situação oposta à zircônia aconteceu com a liga metaloceramica Fit Cast SB. Na tentativa de melhorar a aderência do compósito às ligas, de acordo com Di Francescantonio et $a l .{ }^{19}$, existem muitos métodos como tratamento eletrolítico, condicionamentos químicos, aplicação de primer adesivo, e métodos de cobertura com sílica das ligas metálicas. Para este estudo, foi utilizado o Metal/ Zirconia Primer para a liga, que mostrou uma resistência de união similar entre o grupo controle FF e o auto-aderente DF, sem diferença estatística nos tempos de uma semana e um ano isoladamente. O jateamento com partículas de óxido de alumínio removeu a camada contaminada e criou uma superfície rugosa que proporcionou a união com os compósitos fluidos, bem como uma grande área de união na superfície. Tem sido relatado que o processo de jateamento pode formar uma película passiva feita de $\mathrm{Ni}, \mathrm{Cr}$ e óxidos de Co, segundo os resultados de Yoshida et al. ${ }^{20}$.

O Metal/Zirconia Primer, como já descrito, é constituído por acrilato de ácido fosfônico. O fabricante descreve que é adequado para as cerâmicas com óxidos e todos o tipos de liga metálicas. Neste estudo, os resultados mostraram a eficiência do Metal/Zirconia Primer na resistência de união entre a liga Ni-Cr e o compósito fluido controle e o auto aderente Dyad Flow, exceção para o período de um ano. Para ensaios de uma semana, os resultados foram semelhantes aos achados de Di Francescantonio et al. ${ }^{19}$, porém eles usaram outros primers e outras ligas para próteses metalocerâmica.

$\mathrm{O}$ ensaio de resistência de união ao cisalhamento tende a produzir falhas coesivas no substrato ${ }^{15,16}$. Nesse estudo, através de microscopia óptica e de acordo com os autores acima, observou-se falhas mistas para o AD e EE, entre falhas coesivas (no cerômero e na cerâmica reforçada com leucita) e falhas adesivas. Para cerâmica reforçada com zircônia e a liga metalocerâmica, observou-se falhas adesivas.

\section{Conclusão}

Após degradação adesiva causada por um ano de armazenamento em água destilada, ambos os compósitos apresentaram resistências de união significativamente inferiores em todos os substratos, com exceção da cerâmica reforçada por zircônia, que apresentou resultado nulo. Recomendam-se mais estudos sobre o mecanismo de ação e longevidade da utilização dos compósitos fluidos como materiais alternativos para as cimentações adesivas.

\section{Referências}

1. Shimada Y, Yamaguchi S, Tagami J. Microshear bond strength of dual-cured resin cement to glass ceramics. Dent Mater 2002;18(5):380-8

2. Bayne SC, Thompson JY, Swift Junior EJ, Stamatiades P, Wilkerson M. A characterization of first-generation flowable composites. J Am Dent Assoc 1998;129:567-77.

3. Bektas OO, Eren D, Akin EG, Akin H. Evaluation of a selfadhering flowable composite in terms of micro-shear bond strength and microleakage. Acta Odontol Scand 2013;71(34):541-6.

4. Fu J, Kakuda S, Pan F, Hoshika S, Ting S, Fukuoka A, et al. Bonding performance of a newly developed step-less all-inone system on dentin. Dent Mater J 2013;32(2):203-11.

5. Juloski J, Goracci C, Rengo C, Giovannetti A, Vichi A, Vulicevic ZR, et al. Enamel and dentin bond strength of new simplified adhesive materials with and without preliminary phosphoric acid-etching. Am J Dent 2012;25(4):239-43.

6. Poitevin A, De Munck J, Van Ende A, Suyama Y, Mine A, 
Peumans M, et al.Bonding effectiveness of self-adhesive composites to dentin and enamel. Dent Mater 2013;29(2):22130 .

7. Vichi A, Margvelashvili M, Goracci C, Papacchini F, Ferrari M. Bonding and sealing ability of a new self-adhering flowable composite resin in class I restorations.Clin Oral Investig 2013;17(6):1497-506.

8. Van Meerbeek B, De Munck J, Yoshida Y, Inoue S, Vargas $\mathrm{M}$, Vijay $\mathrm{P}$, et al. Buonocore memorial lecture. Adhesion to enamel and dentin: Current status and future challenges. Oper Dent 2003;28:215-35.

9. Sano H, Shono T, Sonoda H, Takatsu T, Ciucchi B, Carvalho RM. Relation between surface area for adhesion and tensile bond strength - evaluation of a microtensile bond test. Dent Mater 1994;10(4):236-40.

10. Peumans M, Van Meerbeek B, Lambrechts P, Vanherle G. Porcelain veneers: a review of the literature. J Dent 2000;2893:163-77.

11. Zhang Y, Lawn BR, Rekow ED, Thompson VP. Effect of sandblasting on the long-term performance of dental ceramics. J Biomed Mater Res B Appl Biomater 2004;71(2):381-6.

12. Edris A, al Jabr A, Cooley RL, Barghi NSEM. Evaluation of etch patterns by three etchants on three porcelains. J Prosthet Dent 1990;64(6):734-9.

13. Roulet JF, Söderholm KJ, Longmate J. Effects of treatment and storage conditions on ceramic/composite bond strength. J Dent Res 1995;74(1):381-7.

14. Teixeira EC, Bayne SC, Thompson JY, Ritter AV, Swift EJ. Shear bond strength of self-etching bonding systems in combination with various composites used for repairing aged composites. J Adhes Dent 2005;7(2):159-64.

15. Garcia RN, Goes MF, Giannini M. Effect of water storage on bond strength of self-etching adhesives to dentin. J Contemp Dent Pract 2007;8(7):46-53.

16. Garcia RN, Nascimento RF, Gomes ACR, Giannini M, Miguel LCM, Moon PC. Bond strength of resin cements to leucite-reinforced ceramics. RSBO 2012;9(2):183-9.

17. Blatz MB, Sadan A, Kern M. Resin-ceramic bonding: a review of the literature. J Prosthet Dent 2003;89(3):268-74.

18. Kuriyama S, Terui Y, Higuchi D, Goto D, Hotta Y, Manabe A, et al. Novel fabrication method for zirconia restorations: bonding strength of machinable ceramic to zirconia with resin cements. Dent Mater J 2011:2(3):419-24.

19. Di Francescantonio M, Oliveira MT, Garcia RN, Romanini JC, Silva NR, Giannini M. Bond strength of resin cements to $\mathrm{Co}-\mathrm{Cr}$ and $\mathrm{Ni}-\mathrm{Cr}$ metal alloys using adhesive primers. $\mathrm{J}$ Prosthodont 2010;19(2):125-9.

20. Yoshida Y, Kamada K, Atsuta M. Adhesive primers for bonding cobalt-chromium alloy to resin. J Oral Rehabil 1999;26:475-8. 